\title{
NON-WELLFOUNDED MEREOLOGY
}

\author{
AARON J. COTNOIR \\ Northern Institute of Philosophy, University of Aberdeen \\ ANDREW BACON \\ Oxford University, Magdalen College
}

\begin{abstract}
This paper is a systematic exploration of non-wellfounded mereology. Motivations and applications suggested in the literature are considered. Some are exotic like Borges' ALEPH, and the TRINITY; other examples are less so, like TIME TRAVELING BRICKS, and even Geach's TIBBLES THE CAT. The authors point out that the transitivity of non-wellfounded parthood is inconsistent with extensionality. A non-wellfounded mereology is developed with careful consideration paid to rival notions of supplementation and fusion. Two equivalent axiomatizations are given, and are compared to classical mereology. We provide a class of models with respect to which the non-wellfounded mereology is sound and complete.
\end{abstract}

This paper explores the prospects of non-wellfounded mereology. An order < (in this case proper parthood) on a domain is said to be wellfounded if every nonempty subset of that domain has a $<$-minimal element. We say that $x$ is a $<$-minimal element of a set $S$ if there is no $y$ in $S$ such that $y<x$. Wellfoundedness rules out any infinite descending <-chains. There are atomless mereologies, sometimes called gunky, in which proper parthood chains are all infinite. ${ }^{1}$ This is one interesting and important case of a nonwellfounded mereology. But notice, wellfoundedness also rules out structures in which for some $x, x<x$; likewise, it rules out cases in which there is some $x$ and $y$ such that $x<y$ and $y<x$. That is, wellfoundedness rules out parthood loops. In this paper, we explore a non-wellfounded mereology that allows for both these sorts of parthood loops.

In $\S 1$, we briefly survey some applications for non-wellfounded mereology that have been suggested in the literature. In $\S 2$, we consider difficulties with the classical definitions of parthood and proper parthood; we discuss extensionality principles in mereology, and argue that extensionality is inconsistent with the transitivity of parthood in certain nonwellfounded scenarios. In $\S 3$, we examine supplementation principles and rival notions of fusion for non-wellfounded mereology. $\$ 4$ examines the relationship between classical mereology and non-wellfounded mereology. We show that the latter is a simple generalization of the former. Finally, we give a class of models for which non-wellfounded mereology is sound and complete in $§ 5$.

\$1. Why? Why would one consider a mereology according to which there could be proper parthood loops? After all, there appears to be a consensus that it is a conceptual truth that parthood is a partial order. Simons (1987) writes,

Received: April 29, 2011.

1 See Tarski (1956) for one formalization. Some philosophers have argued that gunk is metaphysically possible, for example, Sider (1993) and Zimmerman (1996). 
The most obvious formal properties of the [proper] part-relation are its transitivity and asymmetry, from which follows its irreflexivity. [...] These principles are partly constitutive of the meaning of 'part', which means that anyone who seriously disagrees with them has failed to understand the word. (10-11)

Simons' view has been influential; however, there have been dissenters. In this section, we will briefly survey some cases purported to be examples of non-wellfounded objects.

1.1. Exotica. We begin with some of the more exotic cases. Sanford (1993) contends that the naïve notion of parthood does not require antisymmetry. Sanford cites Borges' 'Aleph' as an example: "I saw the Aleph from all points. I saw the earth in the Aleph and in the earth the Aleph once more and the earth in the Aleph [...]." As Sandford notes, the Aleph is intended to have everything as a part, including the earth. But apparently the Aleph is a proper part of the earth, given the fact that it is sitting on the stairs in Beatriz Viterbo's house. van Inwagen (1993) has responded that fictional examples of this sort do not constitute counterevidence to conceptual truths. While Borges may have been knowingly and intentionally writing fiction, other authors have given examples in texts that were not intended as fiction. These examples, however, are no less bizarre.

Strikingly similar to Borges' example, is an interesting passage in the Upanishads (Mascaró, 1965) that outlines the structural relations between Brahman and a 'space' in the heart.

In the center of the castle of Brahman, our own body, there is a small shrine in the form of a lotus-flower, and within can be found a small space. [...] This little space within the heart is as great as this vast universe. The heavens and earth are there, and the sun, and the moon, and the stars; fire and lightning and winds are there; and all that now is and all that is not; for the whole universe is in Him and He dwells within our heart. (120)

Of course, many philosophers would regard such a structure as metaphysically impossible, but it seems an additional question as to whether it is conceptually impossible.

Again, consider another traditional religious doctrine: the Trinity. Abelard (2006) argues that while each person of the Trinity is a part of God, each person of the Trinity is also identical with God, by virtue of having the same essence. Is the Trinity on Abelard's understanding conceptually impossible? Perhaps, but this is not obvious. In any case, given the extraordinary nature of the purported counterexamples, we do not expect many to be convinced. So let us turn now to less exotic, more ordinary examples of parthood loops.

1.2. Puzzles of composition. A standard puzzle of composition concerns a lump of clay that is formed into a statue. One might think it plausible that, although the lump and the statue are not identical, they are completely colocated and 'made up of' exactly the same matter. It is thus highly intuitive to think that they have exactly the same atomic parts. However given a standard mereological 'supplementation' principle, that anything the statue is not a part of is disjoint from some part of the statue, one can infer that the statue is a part of the lump of clay; and by symmetrical reasoning that the lump of clay is a part of the statue. Thus the clay and the statue are parts of each other. But since we are supposing that they are not identical, they must be distinct mutual proper parts. In fact, 


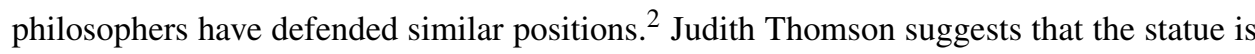
constituted by the clay, and that constitution requires mutual parthood: " $x$ constitutes $y$ at $t$ only if $x$ is part of $y$ at $t$ and $y$ is part of $x$ at $t$ " (Thomson, 1998, p. 155). But constitution, Thomson thinks, does not imply identity. ${ }^{3}$

Another classical puzzle of composition concerns Tibbles the cat. ${ }^{4}$ Tibbles is a cat who has a tail at some time $t_{1}$ and loses it at $t_{2}$. Call Tibbles' tail 'Tail'; and call the part of Tibbles without his tail 'Tib'.

Tib is smaller than Tibbles so they are not identical. But what if we now amputate the cat's tail? Tibbles and Tib will now coincide. If Tibbles is still a cat, it is hard to see by what criterion one could deny that Tib is a cat. [...] But there is just one cat on the mat. So [Tibbles and Tib] cannot be distinct cats. They must be the same cat. (Noonan, 2009)

The literature is filled with many different responses to this puzzle. ${ }^{5}$ Given that Tibbles and Tib have the same proper parts after the amputation it is very natural to infer that they are mutual parts. ${ }^{6}$ Tib might be a proper part of Tibbles before the amputation, but Tibbles and Tib are mutual parts afterwards.

If a non-wellfounded mereology was formally viable, then there are other unexplored responses. One could hold that Tib is a proper part of Tibbles and also that Tib is identical to Tibbles even before amputation. Indeed, Abelard (2006) appeared to have held such a view. ${ }^{7}$

[W] hen a hand has been cut off, that which then stays a human was also staying a human before the amputation, although it was hidden as a part in the human who was the whole. [...] Therefore, although before all amputations there were many parts in one human each of which was human, still there were not for this reason many humans. [...] For the humans here have the same essence. (302)

There may be drawbacks to such approaches; however, these considerations should be weighed against the drawbacks of competing theories. ${ }^{8}$

1.3. Mereology \& time travel. There is a growing literature surrounding the topic of mereology and time travel. ${ }^{9}$ Time travel scenarios have been proposed as counterexamples to certain mereological principles, many of which imply that proper parthood is nonwellfounded.

2 See Cotnoir (2010) for a wellfounded explanation of mutual parthood.

3 See Thomson (1983, p. 208 and p. 219, footnote 12).

4 Originally due to Geach (1962); see also Wiggins (1968).

5 For a good selection, see Rea's (1997) collection.

6 Here one must also appeal to the principle 'Strong Supplementation'—see NWS4 in §3.

7 This view is exactly analogous to his views on the Trinity; indeed, his views on both occur in the same passage.

8 For example, see Eagle (2010), (especially §3), for suggestions of general ways in which the apparently distinguishing characteristics of Tib and Tibbles may be explained away.

9 At the time of writing, the literature includes Gilmore's (2007) argument against perdurantism, Eagle's (2010) response, and Gilmore's (2010) reply. Independently, Effingham \& Robson (2007) have used similar cases to argue against endurantism, Smith's (2009) response, and Effingham's (2010) reply. 
For instance, consider Effingham (2010):

Imagine a cube, with each side measuring $10 \mathrm{~m}$, made of a homogeneous substance. Not only do we take it back to a time that it previously existed at, but we use a shrinking machine and miniaturize by a factor of 100 . We then remove a cube-shaped portion, with edges measuring $10 \mathrm{~cm}$, from the earlier, larger version of the cube and replace that portion with the miniaturized future version (which now fits perfectly). The cube is now a proper part of itself at that time.

Although it is not obvious how such uses of 'proper part' are to be interpreted (as will be discussed in §2), if such time travel stories are to be accepted as legitimate metaphysical possibilities, then one would assume a non-wellfounded mereology is required to model them.

1.4. Other examples. There are other examples of non-wellfounded relations bearing similarities to the parthood relation in the literature. For instance, Kearns (2011) argues that Russellian propositions have mereological parts and that such a mereology must thus be non-wellfounded to account for propositions like the following:

$P$ is abstract.

Here $P$ is the proposition expressed by the above sentence (assuming a proposition is expressed). Indeed, the existence of such propositions is a central component to the solution of the liar paradox defended by Barwise \& Etchemendy (1987). It should be noted that this potential application appears to rely on a presupposed relationship between the constituents of a proposition and the parts of a proposition. We have reservations about the similarities between these notions, and so are unsure that the non-wellfounded mereology advanced below has application here. ${ }^{10}$

Another example can be drawn from the metaphysics of sets. Lewis (1991) has argued that sets themselves may have nontrivial mereological properties. Although not the Lewisian view, if a set's members are among its parts, then one must countenance a nonwellfounded mereology to account for the mereological properties of non-wellfounded sets. Varzi (2006) suggests this application, and concludes,

In view of certain developments in non-wellfounded set theory [...], one might indeed suggest building mereology on the basis of an equally less restrictive notion of parthood that allows for closed loops. This is particularly significant in view of the possibility of reformulating set theory itself in mereological terms [...]. Thus, in this case there is legitimate concern that one of the obvious meaning postulates for 'part' is in fact too restrictive. At present, however, no systematic study of nonwellfounded mereology has been put forward in the literature.

The relationship between set membership and parthood Varzi is suggesting is controversial. However, before we can even begin a systematic investigation of these issues we need to develop a theory of parthood that allows for non-wellfoundedness. But given that

10 In fact, the authors have conflicting opinions. One of us (Bacon) believes the relation that holds between a Russellian proposition and its constituents is not parthood, and hence rejects that the application can be made. The other (Cotnoir) has no settled view, and leaves the possibility of application open. 
no study of non-wellfounded mereology has been undertaken, this paper is an attempt to fill that gap. So, we move from potential applications of the theory to the theory itself.

\$2. Primitives and definitions. What we want, then, is a mereology of objects that may be proper parts of each other, and objects that may be proper parts of themselves. According to classical mereology, parthood is taken to be primitive and is a partial order.

MA1 Reflexivity: $x \leq x$

MA2 Antisymmetry: $(x \leq y \wedge y \leq x) \rightarrow x=y$

MA3 Transitivity: $(x \leq y \wedge y \leq z) \rightarrow x \leq z$

We then go on to define proper parthood in one of two ways:

PP1 $x<y:=(x \leq y) \wedge(x \neq y)$

PP2 $x<y:=(x \leq y) \wedge(y \not \leq x)$.

These axioms ensure that proper parthood does not allow for proper parthood loops. More specifically, assuming MA2, PP1 and PP2 are equivalent. But PP1 rules out any cases where $x<x$. And PP2 rules out any cases where $x<y$ and $y<x$. In effect, proper parthood is a strict order, with no parthood loops.

One option is simply to reject the antisymmetry axiom (MA2), and stipulate that parthood is merely a preorder. ${ }^{11}$ So, PP1 and PP2 are no longer logically equivalent. In fact, in the absence of antisymmetry, it is consistent with PP1 for there to be cases where $x<y$ and $y<x$. However, PP1 still rules out cases where $x<x$ by definition. The relation defined by PP1 is thus nontransitive. On the other hand, PP2 rules out both cases where $x<x$ and cases where $x<y$ and $y<x$. If we want to model both cases, we need different axioms and definitions.

One easy way to construct a non-wellfounded mereology of the desired sort would be to start with a primitive proper parthood relation $<$ and require only that it be transitive.

NWA1 Transitivity: $(x<y \wedge y<z) \rightarrow x<z$.

NWA1 allows for proper parthood loops in both of the desired ways: we will have cases where $x<x$ and cases where $x<y$ while $y<x$. We can then introduce definitions of overlap and parthood in the usual fashion

$\mathbf{P} x \leq y:=x<y \vee x=y$

O $x \circ y:=\exists z(z \leq x \wedge z \leq y)$

Parthood according to $\mathrm{P}$ is reflexive. Moreover, $\leq$ inherits transitivity from the transitivity of $<$ and $=$. However, we shall see that $\leq$ is not in general antisymmetric.

One might wonder what conditions suffice for two composite objects to be identical. Here are some natural candidates:

$$
\begin{aligned}
& x \leq y \wedge y \leq x \\
& \forall z(z<x \leftrightarrow z<y) \\
& \forall z(z \circ x \leftrightarrow z \circ y)
\end{aligned}
$$

In classical mereology, each of these conditions suffice for the identity of $x$ and $y$. These conditions correspond to three so-called extensionality principles.

11 This is the strategy employed in Cotnoir (2010). 
EP Extensionality of Parts: $(x \leq y \wedge y \leq x) \rightarrow x=y$

EPP Extensionality of Proper Parts: $(\operatorname{Co}(x) \vee \operatorname{Co}(y)) \rightarrow(\forall z(z<x \leftrightarrow z<y) \rightarrow x=y)$

EO Extensionality of Overlap: $\forall z(z \circ x \leftrightarrow z \circ y) \rightarrow x=y$

Notice that EP is just the antisymmetry principle (MA2). In EPP, we say an object $x$ is composite if it has a proper part; this is expressed via a predicate $\operatorname{Co}(x):=\exists y(y<x)$. The reason we require that EPP be restricted only to composite objects is to allow for more than one atom. ${ }^{12}$ In EO, it is claimed that any two objects with the same overlappers are identical.

Given the transitivity of $<$ and the presence of distinct mutual parts, we cannot consistently have any of the above extensionality principles. For EP, consider two distinct but mutual proper parts $a$ and $b$. Since $a<b, b<a$, and $a \neq b$, we have $a \leq b$ and $b \leq a$ without $a$ and $b$ being identical. Thus $\leq$ is not antisymmetric; EP fails. For EPP and EO, consider $a$ and $b$ again. By transitivity (NWA1), we have $a<a$ and $b<b$. Moreover, transitivity guarantees that any proper part of $a$ is a proper part of $b$, and vice versa.

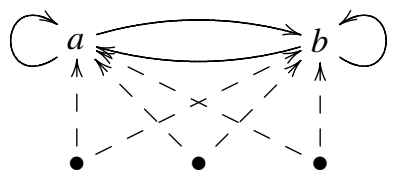

This means that no matter what proper parts $a$ and $b$ have, mutual parthood and transitivity force every part of one to be part of the other. But since $a$ and $b$ have proper parts, they are not atoms; hence, by EPP, $a=b$. But this contradicts our supposition that $a \neq b$. By similar reasoning one can see that $a$ and $b$ have the same overlappers. If we had EO, we could once again infer that $a=b$, contradicting the supposition that $a$ and $b$ are distinct but mutual parts. In effect, transitivity plus extensionality rules out parthood loops.

§3. Axioms for non-wellfounded mereology. In this section, we will explore various supplementation principles and fusion principles for non-wellfounded mereology. We argue that fusions are best understood in one of two ways depending on which supplementation axiom is accepted. In neither case are fusions unique.

3.1. Decomposition. Supplementation principles in mereology are designed to satisfy the intuition that if an object has one proper part, it must have another. Simons (1987) considers a number of such principles, as does Varzi (2006); so, we will follow their lead.

First, consider the straightforward intuition:

NWS1 Weak Company: $x<y \rightarrow \exists z(z<y \wedge z \neq x)$.

NWS1 states that if an object has a proper part, then it has another distinct proper part. This supplementation principle, of course rules out any object $a$ such that $a<a$ and $a$ has no other proper parts.

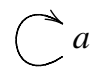

But this is arguably the most basic case of a non-wellfounded object (sometimes called a 'Quine atom'). ${ }^{13}$ And so NWS1 will not do.

12 The same restriction is required on the extensionality principle in set theory with urelements.

13 This name is presumably from the characterization of 'individuals' in Quine's (1980) New Foundations. 
Consider another candidate supplementation principle.

NWS2 Strong Company: $x<y \rightarrow \exists z(z<y \wedge z \not \leq x)$.

Of course, NWS2 is strictly stronger than NWS1, and so inherits all its problems (such as ruling out the Quine atom.) But it is worth seeing that NWS2 rules out all cases of mutual parthood. For if $a<b$ and $b<a$, then by transitivity, we have it that $a<a$ and $b<b$. Moreover, any $z$ that is part of $a$ will be part of $b$, and vice versa. But then there can be no part of $a$ that is not a part of $b$; there can be no witness for $z$ in NWS2. Thus all models like the one below are ruled out.

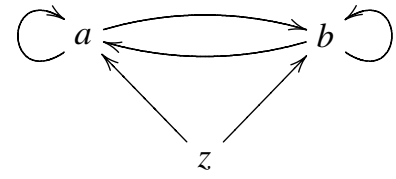

But this, like extensionality, ruins the whole motive for non-wellfounded parthood.

Most mereologists have accepted the next principle $;{ }^{14}$ some have gone so far as to claim that it is constitutive of the concept of parthood. ${ }^{15}$

NWS3 Weak Supplementation: $x<y \rightarrow \exists z(z \leq y \wedge \neg z \circ x)$.

But NWS3 is also inconsistent with any proper parthood relation that is not irreflexive and asymmetric. Unlike NWS1, NWS3 rules out any reflexive proper parthood structure at all, even if that object has other proper parts. Assume $a<a$. Assume also that there are $b$ and $c$ s.t. $b<a$ and $c<a$.

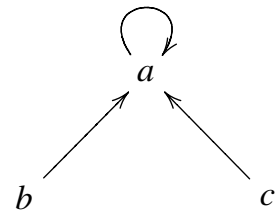

There is still no $z \leq a$ such that $\neg z \circ a$, simply by definition.

There is, however, a principle which is seemingly stronger than NWS3 which is consistent with non-wellfoundedness in this setting.

NWS4 Strong Supplementation: $y \nless \leq x \rightarrow \exists z(z \leq y \wedge \neg z \circ x)$.

Given MA1-MA3 and PP1 or PP2, NWS4 implies NWS3. However, that proof relies on the antisymmetry of $\leq$, which fails here. Notice, the antecedent in the supplementation principle is $y \not \leq x$, which implies that $y \nless x$ and $y \neq x$. This is actually a weaker constraint than $x<y$, the antecedent in NWS1-NWS3. NWS4 does not rule out all reflexive parts, nor does it rule out all mutual parthood structures. It does, however, rule out certain models, like the following.

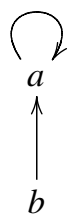

In this case, we have a reflexive part $a$ with only one other proper part $b$. But $a \not \leq b$ and there is no part of $a$ that does not overlap $b$. If the above model included $c<a$ but

14 For some rare exceptions, see Forrest (2002) and Smith (2009).

15 See Simons (1987) and Varzi (2009). 
$c \nless b$ and $b \nless c$ (viz. the countermodel to NWS3), it would be a model of NWS4. In fact, this principle does seem to restrict our models to the sorts we would like, by requiring a 'remainder'.

In in the presence of antisymmetry, NWS4 implies the extensionality of overlap (EO) and proper parthood (EPP). ${ }^{16}$ However, as we shall see in $\$ 4.2$, in the non-wellfounded setting NWS4 does not imply any extensionality principles. The following nonextensional model satisfies NWS4.

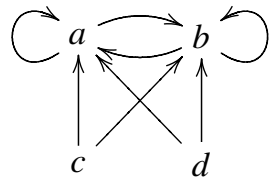

Because NWS4 captures the 'remainder' intuition while still being consistent with nonwellfounded objects like Quine atoms and mutual proper parts, there is good prima facie reason to accept it as an axiom. It may seem a bit surprising that the axiom that guarantees extensionality in the classical setting is consistent with nonextensional non-wellfounded mereology. However, that guarantee relies crucially on the antisymmetry of $\leq$, which fails here.

There is another candidate for supplementation that is worth considering.

NWS5 Complementation: $y \not \leq x \rightarrow \exists z \forall w(w \leq z \leftrightarrow(w \leq y \wedge \neg w \circ x))$.

This principle is a lot like NWS4, with the exception that instead of stipulating the existence of some non- $x$-overlapping remainder of $y$, it stipulates the existence of a remainder of $y$ that consists of (or is composed of) all and only the parts of $y$ disjoint from $x$. Built in to NWS5 is a principle regarding composition. Consider the following example:

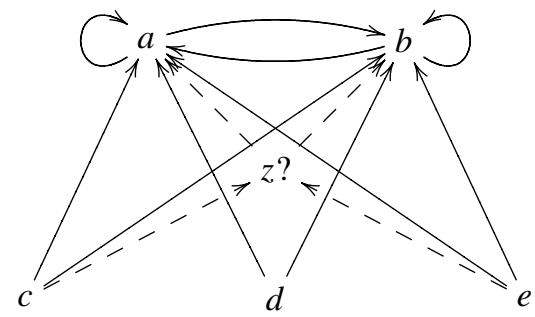

In this case, $a$ and $b$ are mutual parts who share $c, d, e$ as proper parts. The question here is this: must $c$ and $e$ have an upper bound $z$ which does not have $d$ as a part? NWS4 does not require this, while NWS5 does. If there is no such $z$, then this model is ruled out by complementation. Varzi (2006) rightly suggests that NWS5 bleeds into principles regarding composition:

As it turns out, the extra strength of [NWS5] is therefore best appreciated in terms of the sort of mereological aggregates that this principle would force us to accept, aggregates that are composed of two or more parts of a given whole. This suggests that any additional misgivings about [NWS5] $[\ldots]$ are truly misgivings about matters of composition.

Thus, we will withhold judgment on NWS5 until we examine various fusion axioms.

16 Strong supplementation is generally thought to be responsible for extensionality principles in mereology. See Simons (1987, pp. 28-29), and Casati \& Varzi (1999, p. 40). 
3.2. Composition. What sort of fusions are appropriate to a non-wellfounded mereology? We begin with three candidate definitions of fusion. Here, we say that $t$ is a fusion of the $\varphi$ 's, where $\varphi$ is any predicate without $t$ free and $t$ is a term. ${ }^{17}$

F1 $\mathrm{Fu}_{1}(t, \varphi):=\forall y(y \circ t \leftrightarrow \exists x(\varphi \wedge y \circ x))$

F2 $\mathrm{Fu}_{2}(t, \varphi):=\forall x(\varphi \rightarrow x \leq t) \wedge \forall y(y \leq t \rightarrow \exists x(\varphi \wedge y \circ x))$

F3 $\mathrm{Fu}_{3}(t, \varphi):=\forall x(\varphi \rightarrow x \leq t) \wedge \forall y \forall x((\varphi \rightarrow x \leq y) \rightarrow t \leq y)$

F1 says that $t$ is the fusion of the $\varphi$ s when $t$ overlaps exactly those things that overlap a $\varphi$. $\mathrm{F} 2$ says that $t$ is the fusion of the $\varphi$ s when any $\varphi$ is part of $t$ and any part of $t$ overlaps some $\varphi$. F3 says that $t$ is the fusion of the $\varphi$ s when $t$ has all the $\varphi \mathrm{s}$ as parts and $t$ is part of any other $y$ that has all the $\varphi s$ as parts. NWF3 is usually thought of as defining the notion of a least upper bound. However, since we do not have the antisymmetry of $\leq$, it really only expresses the notion of minimal upper bounds. ${ }^{18}$

Corresponding to each type of fusion, there are axiom schemes which assert the existence of each type of fusion for every formula $\varphi$ without $z$ free.

NWE1 $\exists x \varphi \rightarrow \exists z \mathrm{Fu}_{1}(z, \varphi)$

NWE2 $\exists x \varphi \rightarrow \exists z \mathrm{Fu}_{2}(z, \varphi)$

NWE3 $\exists x \varphi \rightarrow \exists z \mathrm{Fu}_{3}(z, \varphi)$

All three definitions of fusion (NWF1-NWF3) are equivalent in the context of any axiomatization that yields classical mereology. Which fusion existence axiom (NWE1-NWE3) yields classical mereology depends on the choice of other axioms. In nonclassical mereologies, the definitions of fusion are often not equivalent. In the presence of transitivity (NWA1) only, we can prove that $\forall z\left(\mathrm{Fu}_{2}(z, \varphi) \rightarrow \mathrm{Fu}_{1}(z, \varphi)\right)$, but not vice versa. So, in one way, NWE2 is stronger than NWE1. The following model validates NWA1 and NWE1 but not NWE2:

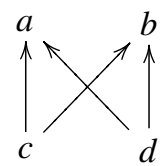

Do $a$ and $b$ have a fusion? It might appear not. There is no object 'made up' of $a$ and $b$ together. However, it turns out that $a$ is a fusion (in the sense defined by $\mathrm{Fu}_{1}$ ) of $a$ and $b$. Let the extension of $\varphi$ here be just $a$ and $b$. Note that every object overlaps $a$ if and only if it overlaps $b$. Hence, every object overlaps $a$ if and only if there's some $\varphi$ that it overlaps. Moreover, $b$ is also a fusion 1 of $a$ and $b .{ }^{19}$ On the other hand, $a$ and $b$ do not have a fusion 2 in this model since there is nothing of which both $a$ and $b$ are a part, preventing the first conjunct of NWF2 being satisfied.

It should be noted, however, that in the presence of strong supplementation (NWS4) and transitivity (NWA1) these two notions of fusion are equivalent. Firstly, we note that the principle $\forall z(z \circ y \rightarrow z \circ x) \rightarrow y \leq x$ is a straightforward consequence of NWS4. Suppose, now, that $x$ is a fusion ${ }_{1}$ of the $\varphi$ s so that (*): $\forall y(x \circ y \leftrightarrow \exists z(\varphi(z) \wedge z \circ y))$. We then

17 These definitions of fusions largely follow those discussed in Hovda (2009), where there he calls them 'Type-1', 'Type-2', and 'Mub', respectively. They also correspond to the definitions of fusion in Varzi (2006), where he calls their corresponding existence axioms 'Strong Sum', 'Strong $\operatorname{Sum}_{b}$ ', and 'Strong $\operatorname{Sum}_{a}$ ', respectively.

18 See Hovda (2009, §1.3.1).

19 This point was first made in Hovda (2009). 
want to show $x$ is a fusion 2 of the $\varphi$ s- that is, we want to show (1) that $\forall y(\varphi(y) \rightarrow y \leq x)$ and (2) that $y \leq x \rightarrow \exists z(\varphi(z) \wedge z \circ y)$. (2) is immediate from (*). For (1) suppose that $\varphi(y)$ and let $z \circ y$. Since $z$ overlaps a $\varphi$ it follows, by $(*)$, that $z$ overlaps $\mathrm{x}, z \circ x$. By the principle $\forall z(z \circ y \rightarrow z \circ x) \rightarrow y \leq x$, we have shown that $y \leq x$ as required for (1).

Consider also the model ruled out by complementation NWS5:

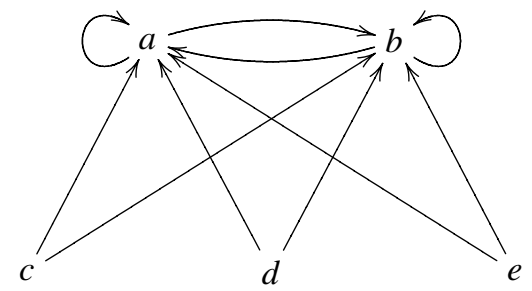

According to $\mathrm{Fu}_{2}$, neither $a$ nor $b$ count as a fusion of $c$ and $e$. And this model will be ruled out by NWE2, since there is no fusion for $c$ and $e$. Intuitively, it does seem as though the model is missing something. It appears that you can simply fuse together $c$ and $e$ to get all of $a$ (or $b$ for that matter) including $d$, even though neither $c$ nor $e$ overlap with $d$. And that seems to be problematic with our intuitive notion of mereological sum.

This is precisely the reason that minimal upper bounds, as defined by $\mathrm{Fu}_{3}$, are problematic. ${ }^{20}$ For according to $\mathrm{Fu}_{3}, a$ (and for that matter $b$ ) does count as a fusion of $c$ and $e$, even though $a$ has a completely unrelated part, $d$. There are some who are attracted to this notion of fusion. ${ }^{21}$ However, Varzi (2009) argues against such a notion of fusion:

All this is hard to swallow. No matter how exactly one defines the word, surely a fusion is supposed to be entirely composed of the things it fuses.

We think that Varzi is right, but that this criticism only applies in contexts where complementation (NWS5) fails. Recall that the complementation axiom rules out such models and requires the existence of an upper bound of $c$ and $e$ that does not have $d$ as a part. So, there is reason to think that NWE3 is an adequate fusion axiom scheme only when paired with NWS5.

As mentioned above, $\mathrm{Fu}_{3}$ is usually associated with least upper bounds, a standard algebraic notion of fusion. However, without antisymmetry, we do not have least upper bounds because we do not, in general, have the uniqueness of fusions. In fact, on neither $\mathrm{F} 1, \mathrm{~F} 2$, nor F3 do we have the uniqueness of fusions.
UF1 $\left(\mathrm{Fu}_{1}(x, \varphi) \wedge \mathrm{Fu}_{1}(y, \varphi)\right) \rightarrow(x=y)$
UF2 $\left(\mathrm{Fu}_{2}(x, \varphi) \wedge \mathrm{Fu}_{2}(y, \varphi)\right) \rightarrow(x=y)$
UF3 $\left(\mathrm{Fu}_{3}(x, \varphi) \wedge \mathrm{Fu}_{3}(y, \varphi)\right) \rightarrow(x=y)$

For example, consider the pair of mutual parts $a$ and $b$, neither of which have any other proper parts.

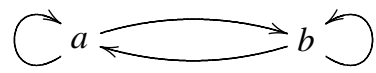

Let the extension of $\varphi$ be $\{a, b\}$. Then for each $i=1,2,3$ we have both $\operatorname{Fu}_{i}(a, \varphi)$ and $\mathrm{Fu}_{i}(b, \varphi)$. Adding further elements to the model containing $a$ and $b$ as parts would only introduce more fusions if anything. It seems clear that in the presence of non-wellfoundedness like that above none of the fusions considered can be unique.

20 See Varzi (2009).

21 See Hovda (2009), Sharvey (1983), and Link (1983). 
So, there are two options that best axiomatize non-wellfounded mereology.

$\mathbf{P} x \leq y:=x<y \vee x=y$

O $x \circ y:=\exists z(z \leq x \wedge z \leq y)$

F1 $\operatorname{Fu}_{1}(t, \varphi):=\forall y(y \circ t \leftrightarrow \exists x(\varphi \wedge y \circ x))$

NWA1 $(x<y \wedge y<z) \rightarrow x<z$

NWS4 $y \not \leq x \rightarrow \exists z(z \leq y \wedge \neg z \circ x)$

NWE1 $\exists x \varphi \rightarrow \exists z \mathrm{Fu}_{1}(z, \varphi)$

NWA1 + NWS4 + NWE1 is an axiomatization of non-wellfounded mereology. Note that in the presence of strong supplementation the notions of a fusion 1 and a fusion amount to $^{2}$ the same thing, so NWE2 is derivable in this system. Alternatively:

$\mathbf{P} x \leq y:=x<y \vee x=y$

O $x \circ y:=\exists z(z \leq x \wedge z \leq y)$

F3 $\mathrm{Fu}_{3}(t, \varphi):=\forall x(\varphi \rightarrow x \leq t) \wedge \forall y(\forall x(\varphi \rightarrow x \leq y) \rightarrow t \leq y)$

NWA1 $(x<y \wedge y<z) \rightarrow x<z$

NWS5 $y \not \leq x \rightarrow \exists z \forall w(w \leq z \leftrightarrow(w \leq y \wedge \neg w \circ x))$

NWE3 $\exists x \varphi \rightarrow \exists z \mathrm{Fu}_{3}(z, \varphi)$

NWA1 + NWS5 + NWE3 is an axiomatization of non-wellfounded mereology, ${ }^{22}$ in fact, the two axiomatizations result in the same non-wellfounded mereology. ${ }^{23}$

§4. Classical and non-wellfounded mereology. We now turn to a comparison between classical and non-wellfounded mereology. Given the failure of many of the classical supplementation, extensionality, and fusion uniqueness principles one might be led into thinking that there is little if any parallel to be drawn between the system we discussed in the previous section and classical extensional mereology. In $\$ 4.1$, we show the connection is quite simple: one needs only to drop the asymmetry of proper parthood from a standard axiomatization of classical mereology. In $\S 4.2$, we examine exactly how much extensionality non-wellfounded mereology contains.

4.1. Classical mereology. Classical mereology is usually axiomatized with a primitive improper parthood relation. Perhaps the most common axiomatization has $\leq$ as a partial order, strong supplementation, and the existence of fusion ${ }_{1} \mathrm{~s}$, all stated using the primitive $\leq$ and with overlap defined by $\mathrm{O}$.

MA1 $x \leq x$

MA2 $(x \leq y \wedge y \leq x) \rightarrow x=y$

MA3 $(x \leq y \wedge y \leq z) \rightarrow x \leq z$

NWS4 $y \not \leq x \rightarrow \exists z(z \leq y \wedge \neg z \circ x)$

NWE1 $\exists z \varphi \rightarrow \exists x \forall y(x \circ y \leftrightarrow \exists z(\varphi \wedge y \circ z))$

With only a minor change we can formulate classical mereology with a primitive proper parthood relation instead:

NWA1 $(x<y \wedge y<z) \rightarrow x<z$

NWA2 $x<y \rightarrow y \nless x$

22 Relatedly, see the wellfounded but nonextensional mereology axiomatized in Cotnoir (2010); it has identical axioms but a different primitive and altered definitions.

23 Thanks to Paul Hovda for discussion. 
NWS4 $y \not \leq x \rightarrow \exists z(z \leq y \wedge \neg z \circ x)$

NWE1 $\exists z \varphi \rightarrow \exists x \forall y(x \circ y \leftrightarrow \exists z(\varphi \wedge y \circ z))$

Here again $\leq$ and $\circ$ are defined according to P and O. NWA1 and NWA2 are the axioms for a strict partial order and and MA1-MA3 are axioms for a weak partial order. Other than that, the systems are identical modulo definitions. It is well-known that every strict partial order determines a weak partial order and vice versa. So, NWA1 + NWA2 + NWS4 + NWE1 is equivalent to classical mereology.

The only principle that obviously rules out non-wellfoundedness is the asymmetry principle NWA2. NWA2 obviously rules out cases of mutual parthood between distinct objects. But it also rules out cases of self-parthood (e.g., the Quine atom), since an instance of NWA2 is $a<a \rightarrow a \nless a$, and therefore $a \nless a$. Dropping NWA2 leaves us with precisely the non-wellfounded mereology discussed in $\S 3$.

4.2. Extensionality. Recall that one of the primary points of contrast between classical and non-wellfounded mereology is that one does not seem to have any form of extensionality in non-wellfounded mereology. In particular, one cannot maintain the following extensionality principles:

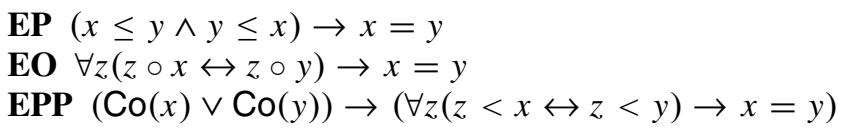

The failure of antisymmetry (EP) is hardly surprising. Indeed, one promising application of non-wellfounded mereology is to model exactly the kind of phenomena that EO rules out. For example, the paradoxes of material constitution have lead people to reject EO and EPP on independent grounds. ${ }^{24}$ It is perhaps surprising, however, that the other cases of non-wellfoundedness discussed in $\S 1$ would require giving up EO and EPP as well.

On the other hand, it can be seen that all the failures of extensionality stem from failures of EP. ${ }^{25}$ Indeed, classical extensional mereology can prove the following extensionality principles without the use of NWA2:

EO* $\forall z(z \circ x \leftrightarrow z \circ y) \rightarrow(x \leq y \wedge y \leq x)$

EPP* $(\mathrm{Co}(x) \vee \mathrm{Co}(y)) \rightarrow(\forall z(z<x \leftrightarrow z<y) \rightarrow(x \leq y \wedge y \leq x))$

According to these weakened extensionality principles, any two (composite) objects with the same overlappers or proper parts are mutual improper parts. Then, in classical mereology, we reason by antisymmetry (EP) to conclude that any such objects are identical. It is only this last step that is not allowed in non-wellfounded mereology.

Similarly we may also obtain weakened versions of fusion uniqueness:

UF1* $\left(\mathrm{Fu}_{1}(x, \varphi) \wedge \mathrm{Fu}_{1}(y, \varphi)\right) \rightarrow(y \leq x \wedge x \leq y)$

UF2* $\left(\mathrm{Fu}_{2}(x, \varphi) \wedge \mathrm{Fu}_{2}(y, \varphi)\right) \rightarrow(y \leq x \wedge x \leq y)$

UF3* $\left(\mathrm{Fu}_{3}(x, \varphi) \wedge \mathrm{Fu}_{3}(y, \varphi)\right) \rightarrow(y \leq x \wedge x \leq y)$

As before we may prove each of UF ${ }^{*}-\mathrm{UF} 3 *$ in classical extensional mereology without the use of NWA2. It is only because we cannot infer identity from mutual parthood that we cannot get the full strength of uniqueness.

24 See for example, Hawthorne (2006), and papers in Rea (1997).

25 See also Cotnoir (2010) for further discussion of the connection between antisymmetry and extensionality. 
§5. Models. The aim of this section is to describe a class of models for non-wellfounded mereology. The analogous class in the case of classical extensional mereology is the class of complete Boolean algebras without a least element. We show that a version of the mereology in $\$ 3$ in second-order logic is sound and complete, relative to full secondorder consequence, with respect to these models. In this section, we proceed in a more streamlined fashion; some familiarity with lattice theory and Boolean algebras is assumed in what follows.

It should be noted that even the classical extensional mereology discussed in $\S 4.1$ has unintended models. Since it is a first-order theory with infinite models, it has a countably infinite model. However there are no countably infinite complete Boolean algebras, and thus no countably infinite complete Boolean algebras without a least element. It is natural, then, to work in this context with a second-order fusion axiom:

SO-NWE1 $\forall F(\exists x F x \rightarrow \exists x \forall y(x \circ y \leftrightarrow \exists z(F z \wedge y \circ z)))$

In order to make the comparison with classical extensional mereology we shall look first at its models.

Definition 5.1 Let $\mathcal{X}$ be a nonempty set of subsets of $Y, \mathcal{X} \subseteq \mathcal{P}(Y)$. $\mathcal{X}$ is a field of sets iff $\mathcal{X}$ is closed under complements in $Y$ and finite intersections. A partial order $\langle B, \leq\rangle$ is a Boolean algebra iff it is order isomorphic to a field of sets; that is, there is some bijection, $\sigma: B \rightarrow \mathcal{X}$ with $x \leq y$ iff $\sigma(x) \subseteq \sigma(y)$ for each $x, y \in B .\langle B, \leq\rangle$ is said to be complete iff every subset of $B$ has a supremum.

From our definition it follows that $\emptyset \in \mathcal{X}$ for every field of sets $\mathcal{X} \subseteq \mathcal{P}(Y)$, since if $X \in \mathcal{X}, Y \backslash X \in \mathcal{X}$ and thus $X \cap(Y \backslash X) \in \mathcal{X}$. Thus every Boolean algebra has a bottom element—an element below every other element. This makes Boolean algebras unsuitable models of mereology.

Definition 5.2 A quasi-Boolean algebra is a Boolean algebra in which the bottom element has been removed, and the order is restricted to the remaining elements.

$B$ is a complete quasi-Boolean algebra if and only if $B$ is a quasi-Boolean algebra and every nonempty subset of $B$ has a supremum in $B$.

The definition of a complete quasi-Boolean algebra is much like that of a complete Boolean algebra except that, since we do not have a bottom element, the empty set has no supremum. It is well-known that second-order classical extensional mereology is sound and complete with respect to the class of complete quasi-Boolean algebras, and that furthermore every full second-order model of classical mereology is a complete quasi-Boolean algebra. $^{26}$

In order to characterize non-wellfounded mereology, we need a class of models that play a role analogous to that of complete quasi-Boolean algebras for classical extensional mereology. The first thing to account for is that non-wellfounded mereology takes proper parthood as a primitive. Unlike in the classical case, proper and improper parthood are not interdefinable. In particular, one can have several distinct proper parthood relations that give rise to the same improper parthood relation. Let us make this precise:

DEFINITION 5.3 For any relation $R$ over domain $D$ (i.e., for any $R \subseteq D \times D$ ) the reflexive closure of $R$ in $D$ is $R \cup\{\langle x, x\rangle \mid x \in D\}$.

26 The point is originally due to Tarski (1956). See also Niebergall (2009). 
Let $S$ be a reflexive relation over the domain $D$. Then we say that $R$ is a proper parthood relation for $S$ in $D$ if and only if $S$ is the reflexive closure of $R$ in $D$.

Given any reflexive relation $S$ on $D$ (an improper parthood relation), there will always be a largest proper parthood relation for $S$ in $D$ (namely, $S$ itself), and a smallest (namely, $S \backslash\{\langle x, x\rangle \mid x \in D\}$ ). The former case corresponds to a scenario where every element is a proper part of itself, the latter to a scenario where nothing is a proper part of itself. However, the latter relation is not always transitive when $S$ is. In what follows, we restrict our attention to transitive proper parthood relations for a given improper parthood relation in a domain.

We suggest the following class of 'non-wellfounded algebras'.

DEFINITION 5.4 A non-wellfounded algebra is a quintuple $\langle A, B, \leq, \sqsubset, 1\rangle$ where

- A is a complete quasi-Boolean algebra with order $\leq$

- $A \subseteq B$

- ' $: B \rightarrow A$ where $a^{\prime}=$ a for every $a \in A$

- $\sqsubset$ is a transitive proper parthood relation for the relation $\left\{\langle x, y\rangle \in B \times B \mid x^{\prime} \leq y^{\prime}\right\}$ in $B$.

Intuitively, $A \subseteq B$ is the wellfounded part of the universe. The wellfounded part will always be a complete quasi-Boolean algebra, so that whenever $A=B$ and $x \sqsubset y$ iff $x^{\prime}<y^{\prime}$ we have classical mereology. That is, non-wellfounded algebras generalize the classical models in a straightforward way.

THEOREM 5.5. Second-order non-wellfounded mereology is sound with respect to non-wellfounded algebras.

Proof. Let $\langle A, B, \leq, \sqsubset, \prime\rangle$ be a non-wellfounded algebra. Define $x \sqsubseteq y:=x \sqsubset y \vee x=$ $y$. Now, let $x \diamond y$ mean that there is some element $z$ in $B$ with $z \sqsubseteq x$ and $z \sqsubseteq y$, and write $x \circ y$ to mean that $x, y \in A$ and for some $z \in A, z \leq x$ and $z \leq y$. It is easy to verify (a) that for any $x, y \in B, x^{\prime} \leq y^{\prime}$ iff $x \sqsubseteq y$ and (b) that for any $x, y \in B, x^{\prime} \circ y^{\prime}$ if and only if $x \diamond y$. For (a) note that for every $x, y \in B, x^{\prime} \leq y^{\prime}$ iff either $x \sqsubset y$ or $x=y$ since $\sqsubset$ is a proper parthood relation for $x^{\prime} \leq y^{\prime}$ in $B$. For (b) note that if $x^{\prime} \circ y^{\prime}$ then for some $z \in A$, $z \leq x^{\prime}$ and $z \leq y^{\prime}$, and thus, since $z=z^{\prime}$, for some $z \in B z \sqsubseteq x$ and $z \sqsubseteq y$ by (a), so $x \diamond y$. Conversely, if $x \diamond y$, then for some $w \in B, w \sqsubseteq x$ and $w \sqsubseteq y$, and thus for some $w \in B$ (by (a) again), $w^{\prime} \leq x^{\prime}$ and $w^{\prime} \leq y^{\prime}$. Finally, note that $w^{\prime} \in A$, so there is some $z \in A$ (namely $w^{\prime}$ ) with $z \leq x^{\prime}$ and $z \leq y^{\prime}$, and since $x^{\prime}, y^{\prime}$ are automatically in $A, x^{\prime} \circ y^{\prime}$.

We can then proceed to show that $\langle A, B, \leq, \sqsubset, \prime\rangle$ models non-wellfounded mereology.

(1) NWA1: we have it that $\sqsubset$ is transitive by definition.

(2) NWS4: Suppose that $y \nsubseteq x$, so that $y^{\prime} \not x^{\prime}$ by (a). Since $A$ is a complete quasiBoolean algebra there is some $z \in A$ with $\neg z \circ x^{\prime}$ and $z \leq y^{\prime}$. Since $z^{\prime}=z$ we have $\neg z^{\prime} \circ x^{\prime}$ and $z^{\prime} \leq y^{\prime}$ and thus $\neg z \diamond x$ and $z \sqsubseteq y$.

(3) SO-NWE1: We shall show that for any nonempty subset $X \subseteq B$ there is an element $x$ of $B$ such that for any $y \in B, x \diamond y$ if and only if $y \diamond z$ for some $z \in X$.

Let $X^{\prime}:=\left\{x^{\prime} \mid x \in X\right\}$. Since $A$ is a complete quasi-Boolean algebra, and $\emptyset \neq X^{\prime} \subseteq A$, there is some $x \in A$ such that the following holds for every $y \in A$.

(*) $x \circ y$ if and only if there is some $z \in X^{\prime}$ with $y \circ z$.

Note that since $x \in A, x^{\prime}=x$. We shall show that $x$ is the required fusion. Let $y \in B$ 
$(\Rightarrow)$ : Suppose that $x \diamond y$. So $x^{\prime} \circ y^{\prime}$, and since $x=x^{\prime}, x \circ y^{\prime}$. By $(*)$ it follows that there is some $w \in X^{\prime}$ such that $y^{\prime} \circ w$. Since $w \in X^{\prime}$, there is some $z \in X$ with $w=z^{\prime}$. So there is some $z \in X$ such that $y^{\prime} \circ z^{\prime}$, that is, $y \diamond z$ as required.

$(\Leftarrow)$ : Suppose $y \diamond z$ for some $z \in X$. Then $z^{\prime} \in X^{\prime}$ so by $(*) y^{\prime} \circ x^{\prime}$-that is, $y \diamond x$.

THEOREM 5.6. Second-order non-wellfounded mereology is complete with respect to non-wellfounded algebras.

Proof. Suppose that $\Gamma$ is a consistent set containing NWA1, NWS4, and SO-NWE1. ${ }^{27}$ Then $\Gamma$ has a standard second-order model, $\langle D, R\rangle$, with domain $D$ and with proper parthood interpreted by $R$. It will be sufficient to show $\langle D, R\rangle$ is equivalent to a nonwellfounded algebra, so that $\Gamma$ is satisfied in some non-wellfounded algebra. Let $S$ be the reflexive closure of $\left(R \cap R^{-1}\right)$ in $D$, and let $A^{*}:=D / S=\left\{[x]_{S} \mid x \in D\right\}$, the set of equivalence classes of $D$ under $S$. $A^{*}$ is a complete quasi-Boolean algebra under the order: $E \leq^{*} F$ iff $E=F$ or, for some $x$ and $y$, with $R x y, x \in E$ and $y \in F .{ }^{28}$ Using the axiom of choice there is a function $f: A^{*} \rightarrow D$ with $f(E) \in E$ for each $E \in A^{*}$. Let $A$ be the range of $f$. $A$ inherits the structure of a complete quasi-Boolean algebra from $A^{*}$ via $f$. Let $\leq$ denote this ordering on $A$, that is, let $f(E) \leq f(F)$ iff $E \leq^{*} F$. For each $x \in D$ let $x^{\prime}=f\left([x]_{S}\right)$. We claim that $\langle A, D, \leq, R, \prime\rangle$ is a non-wellfounded algebra.

To complete the proof we must only show that $R$ is a transitive proper parthood relation for the relation $x^{\prime} \leq y^{\prime}$ in $D$. That is, we must show that $x^{\prime} \leq y^{\prime}$ if and only if $R x y$ or $x=y$ for each $x, y \in D$.

$(\Leftarrow)$. If $a=b$, then $a^{\prime}=b^{\prime}$ so clearly $a^{\prime} \leq b^{\prime}$. Suppose that Rab. So $[a]_{S} \leq^{*}[b]_{S}$, and thus $a^{\prime} \leq b^{\prime}$.

$(\Rightarrow)$. Suppose $a^{\prime} \leq b^{\prime}$. This means that $f\left([a]_{S}\right) \leq f\left([b]_{S}\right)$ so that $[a]_{S} \leq^{*}[b]_{S}$. So either $[a]_{S}=[b]_{S}$, in which case $R a b$ or $a=b$, or there is an $x$ and $y$ with $x \in[a]_{S}, R x y$ and $y \in[b]_{S}$. In the latter case we have (1) either $a=x$ or $R a x$; (2) $R x y$; and (3) either $y=b$ or $R y b$. In each of these situations we get $R a b$ by transitivity.

Although non-wellfounded algebras are versatile enough to capture every possible nonwellfounded scenario consistent with the axioms of non-wellfounded mereology, one might wonder what heuristic value they have. Intuitively we may think of $A$ as underlying the wellfounded part of the universe. The mereological structure of the whole universe is inherited from the structure of $A$ through their projections into $A$, as determined by the ' function. To allow mutual parthood between distinct elements, $a$ and $b$, we consider $a$ to be a part of $b$ if their projections into $A$ are below each other. Distinct elements in $B$ may be projected to the same element of $A$ allowing for mutual parthood between distinct elements.

Example. To see how these models might be useful let us apply this idea to the problem of persistence described in Geach's TibBles THE CAT. Sider (2003) introduces a

27 'Consistent' here means consistent in second-order logic under the semantic consequence relation.

28 By the equivalences in $\$ 3.2$ it can be seen that $\langle D, R\rangle$ models NWS5 and SO-NWE3, a secondorder version of NWE3. By an argument in Cotnoir (2010) it can be shown that under these assumptions $D / S$ with the order $\leq *$ also models versions of NWS5 and SO-NWE3 in which improper parthood is taken as primitive. Since $D / S$ with the order $\leq^{*}$ is a partial order by construction, it follows that it is second-order model of classical mereology, and is therefore a complete quasi-Boolean algebra. 
notion of 'parthood-at- $t$ ' that is supposed to be neutral vocabulary which both perdurantists and endurantists about persistence could accept. In perdurantist vocabulary, Sider defines parthood-at- $t$ as follows

$x$ is a part of $y$ at $t$ iff $x$ 's instantaneous temporal part at $t$ is a part of $y$ 's instantaneous part at $t$.

Sider's parthood-at- $t$ relation is a non-wellfounded parthood relation. For example, if $t$ is some time after Tibbles' amptutation, Tibbles' and Tib's instantaneous temporal part at $t$ are identical; thus Tibbles and Tib are distinct but mutual parts-at- $t$. Indeed, nonwellfounded mereology is the natural logic of parthood-at- $t$. This follows from the fact that the parthood-at- $t$ model of parthood gives rise to a non-wellfounded algebra: the instantaneous temporal part of $x$ at $t$ is just $x$ 's projection into the lattice of subregions of $t .{ }^{29,30}$

§6. Conclusion. We have given a systematic overview of the prospects for non-wellfounded mereology. Two equivalent axiomatizations for non-wellfounded mereology have resulted from careful attention to the kinds of models they accept and reject. The relation between non-wellfounded mereology and classical mereology turned out to be surprisingly intimate. We have given a class of models which are sound and complete with respect to the (second-order) axiomatizations for non-wellfounded mereology. It might prove useful to put to use for the applications laid out in $\S 1$. Perhaps, there are even more phenomena that might be better explained using a non-wellfounded formal metaphysics. But this remains to be seen.

\$7. Acknowledgments We would like to thank the following people for helpful discussion of these ideas: Ralf Bader, Michael Glanzberg, Paul Hovda, Michael Hughes, Graham Priest, Agustìn Rayo, Greg Restall, Dave Ripley, Reed Solomon, Gabriel Uzquiano, and Zach Weber. Thanks also to Don Baxter, Jc Beall, Marcus Rossberg, and Achille Varzi for comments on earlier drafts. Additional thanks are due to an anonymous referee, whose comments led to many improvements.

\section{BIBLIOGRAPHY}

Abelard, P. (2006). Theologica christiana. In Bosley, R. N., and Tweedale, M. M., editors. Basic Issues in Medieval Philosophy, Chapter V.3. Ontario, CA: Broadview Press, pp. 296-303.

Barwise, J., \& Etchemendy, J. (1987). The Liar. Oxford: Oxford University Press.

29 If $t$ is a time (i.e., a certain kind of maximal three-dimensional subregion of space time), then let $A$ be the subregions of $t$ and $B$ the regions of space time which overlap $t . A$ is a complete quasi-Boolean algebra. Notice also that for any region of space time, $X$, overlapping $t$, there is always a largest subregion of $t$ that is also a subregion of $X: X^{\prime}$, the intersection of $X$ with $t$. Thus the quintuple $\left\langle A, B, \leq, \leq^{\prime}, \prime\right\rangle$, where $\leq$ is the subregion relation restricted to $A$ and $\leq^{\prime}$ is the subregion relation restricted to $B$, is a non-wellfounded algebra.

30 This model seems to be well-suited to a 'plenitudinous' approach to coincidence, in which there are as many objects coincident and mutual parts with Tibbles' as a four-dimensionalist would countenence. For a view of this kind, see Hawthorne (2006). 
Casati, R., \& Varzi, A. C. (1999). Parts and Places: The Structures of Spatial Representation. Cambridge, MA: MIT Press.

Cotnoir, A. J. (2010). Anti-symmetry and non-extensional mereology. The Philosophical Quarterly, 60(239), 396-405.

Eagle, A. (2010). Location and perdurance. Oxford Studies in Metaphysics, 5, 53-94.

Effingham, N. (2010). Mereological explanation and time travel. Australasian Journal of Philosophy, 88, 333-345.

Effingham, N., \& Robson, J. (2007). A mereological challenge to endurantism. Australasian Journal of Philosophy, 85, 633-640.

Forrest, P. (2002). Non-classical mereology and its application to sets. Notre Dame Journal of Formal Logic, 43, 79-94.

Geach, P. T. (1962). Reference and Generality. Ithaca, NY: Cornell University Press.

Gilmore, C. (2007). Time travel, coinciding objects, and persistence. Oxford Studies in Metaphysics, 3, 177-198.

Gilmore, C. (2010). Coinciding objects and duration properties: Reply to Eagle. Oxford Studies in Metaphysics, 5, 95-111.

Hawthorne, J. (2006). Three dimensionalism. Metaphysical Essays, 85-111.

Hovda, P. (2009). What is classical mereology? Journal of Philosophical Logic, 38(1), $55-82$.

Kearns, S. (2011). Can a thing be part of itself? American Philosophical Quarterly, 48(1).

Lewis, D. (1991). Parts of Classes. Oxford, UK: Basil Blackwell.

Link, G. (1983). The logical analysis of plural and mass terms: A lattice-theoretical approach. In Bäuerle, R., et al., editors. Meaning, Use, and Interpretation of Language. Berlin: de Gruyter, pp. 303-323.

Mascaró, J., editor. (1965). Upanishads. London: Penguin.

Niebergall, K. (2009). On 2nd order variants of calculi of individuals. Theoria, 24, 169-202.

Noonan, H. (2009). Identity. In Zalta, E. N., editor. The Stanford Encyclopedia of Philosophy. Winter 2009 edition.

Quine, W. V. (1980). From a Logical Point of View (second edition). Chapter New Foundations for Mathematical Logic. Cambridge, MA: Harvard University Press.

Rea, M., editor. (1997). Material Constitution: A Reader. Lanham, MD: Rowan and Littlefield.

Sanford, D. H. (1993). The problem of the many, many composition questions, and naïve mereology. Noûs, 27(2), 219-228.

Sharvey, R. (1983). Mixtures. Philosophy and Phenomenological Research, 44(2), 227-239.

Sider, T. (1993). van Inwagen and the possibility of gunk. Analysis, 53, 285-289.

Sider, T. (2003). Four-dimensionalism: An Ontology of Persistence and Time. Oxford: Oxford University Press.

Simons, P. M. (1987). Parts: A Study In Ontology. Oxford, UK: Clarendon.

Smith, D. (2009). Mereology without weak supplementation. Australasian Journal of Philosophy, 87, 505-511.

Tarski, A. (1956). Foundations of the geometry of solids. In Corcoran, J., editor. Logic, Semantics, Metamathematics. Indianapolis, IN: Hackett.

Thomson, J. J. (1983). Parthood and identity across time. Journal of Philosophy, 80(4), 201-220.

Thomson, J. J. (1998). The statue and the clay. Noûs, 32(2), 149-173. 
van Inwagen, P. (1993). Naïve mereology, admissible valuations, and other matters. Noûs, 27(2), 229-234.

Varzi, A. (2009). Universalism entails extensionalism. Analysis, 69, 599-604.

Varzi, A. (2006). Mereology. In Zalta, E. N., editor. The Stanford Encyclopedia of Philosophy. Winter.

Wiggins, D. (1968). On being at the same place at the same time. Philosophical Review, 77, 90-95.

Zimmerman, D. (1996). Could extended objects be made out of simple parts? An argument for atomless gunk. Philosophy and Phenomenological Research, 56, 1-29.

\title{
NORTHERN INSTITUTE OF PHILOSOPHY \\ UNIVERSITY OF ABERDEEN \\ OLD BREWERY \\ ABERDEEN \\ AB24 3UB \\ UNITED KINGDOM
}

E-mail: a.cotnoir@abdn.ac.uk

\author{
MAGDALEN COLLEGE \\ OXFORD \\ OX1 4AU \\ UNITED KINGDOM
}

